the same portion which illuminated the clouds directly overhead at the time of observation. The rainbow suffered no diminution of brightness where it was apparently crossed by the fluted shadows, the latter being far away in comparison with the bowproducing raindrops, which, of course, were in sunshine.

I regret that I am unable to send a photograph or sketch of the phenomenon, which was a most beautiful one, and must be of rare occurrence. I have never before seen anything similar, nor have I read anywhere a description of a rainbow after sunset.

Allahabad, India, September 18.

S. A. Hili.

\section{Occurrence of Sterna anglica in Belfast Lough.}

IT may possibly interest some of your ornithological readers to know that towards the end of September a specimen of the gul:-billed tern (Sterna anglica) was shot in Belfast Lough. The bird was placed in the hands of Mr. Darragh, of the Museum of that town, and brought by him to me for determination. On consulting the last edition of "Yarrell," I find that it does not appear to have been previously recorded from Ireland.

Queen's College, Belfast, October 8. ROBERT O. CUNNINGHAM.

\section{MODERN VIEWS OF ELECTRICITY. ${ }^{1}$}

\section{PART II.}

III.

$W^{\mathrm{E}}$

E have now glanced through electro-static pheno mena, and seen that they could be all comprehended and partially explained by supposing electricity to be a fluid of perfect incompressibility-in other words, a liquid-permeating everywhere and everything; and by further supposing that in conducting matter this liquid was capable of free locomotion, but that in insulators and general space it was as it were entangled in some elastic medium or jelly, to strains in which electrostatic actions are due. This medium might be burst, in a disruptive discharge, but easy flow could go on only through channels or holes in it, which therefore were taken to represent conductors; and it was obvious that all flow must take place in closed circuits.

To day I want to consider the circumstances of this flow more particularly: to study, in fact, the second division of our subject (see classification on page 532), viz. Electricity in locomotion.

I use the term "locomotion" in order to eliminate rotation and vibration: it is translation only with which we intend now to concern ourselves.

Consider the modes in which water may be made to move from place to place; there are only two: it may be pumped along pipes, or it may be carried about in jugs. In other words, it may travel through matter, or it may travel with matter. Just so it is with heat also: heat can travel in two ways: it can flow through matter, by what is called "conduction" and it can travel with matter, by what is called "convection." There is no other mode of conveyance of heat. You frequently find it stated that there is a third method, viz. "radiation"; but this is not truly a conveyance of heat at all. Heat generates radiation at one place, and radiation reproduces heat at another; but it is radiation which travels, and not heat. Heat only naturally flows from hot bodies to cold, just as water only naturally flows down hill; but radiation spreads in all directions, without the least attention to where it is going. Heat can only flow one way at any given point, but radiation travels all ways at once. If water were dissociated on one planet into its constituent gases, and if these recombined on another planet, it would not be water which travelled from one to the other, neither would the substance obey the laws of motion of water-water would be destroyed in one place, and repro-

Continued from p. 5ix. duced in another; just so is it with the relation between radiation and heat.

Heat, then, like water, has but two direct modes of conveyance from place to place. For electricity the same is true. Electricity can travel with matter, or it can travel through matter ; by convection or by conduction, but in no other known way.

\section{Conduction in Metals.}

Consider, first, conduction. Connect the poles of a voltaic battery to the two ends of a copper wire, and think of what we call "the current." It is a true flow of electricity among the molecules of the wire. If electricity were a fluid, then it would be a transport of that fluid; if electricity is nothing material, then a current is no material transfer ; but it is certainly a transfer of electricity, whatever electricity may be. Permitting ourselves again the analogy of a liquid, we can picture it flowing through, or among, the molecules of the metal. Does it flow through or between them? Or does it get handed on from one to the next continually? We do not quite know ; but the last supposition is often believed to most nearly represent the probable truth. The flow may be thought of as a perpetual attempt to set up a strain like that in a dielectric, combined with an equally perpetual breaking down of every trace of that strain. If the atoms be conceived as little conductors vibrating about and knocking each other, so as to be easily and completely able to pass on any electric charge they may possess, then, through a medium so constituted, electric conduction could go on much as it does go on in a metal. Each atom would receive a charge from those behind it, and hand it on to those in front of it, and thus may electricity get conveyed along the wire. Do not, however, accept this picture as anything better than a possible mode of reducing conduction to a kind of electrostatics - an interchange of electric charges among a series of conductors. If such a series of vibrating and colliding particles existed, then certainly a charge given to any point would rapidly distribute itself over the whole, and the potential would quickly become uniform; but it by no means follows that the actual process of conduction is anything like this. Certainly it is not the simplest mode of picturing it for ordinary purposes. The easiest and crudest idea is to liken a wire conveying electricity to a pipe full of marbles or sand conveying water ; and for many purposes, though not for all, this crude idea suffices.

Leaving the actual mode of conveyance as unknown, let us review how much is certainly known of the process called conduction.

This much is certainly known :-

(I) That the wire gets heated by the passage of a current.

(2) That no trace of a tendency to reverse discharge or spring back exists.

(3) That the electricity meets with a certain amount of resistance or friction-like obstruction.

(4) That this force of obstruction is accurately proportional to the speed with which the electricity travels through the metal-that is, to the intensity of the current per unit area.

About this last fact a word or two must be said. The amount of electricity conveyed per second across a unit area is called the intensity of current; and experiment proves, what $\mathrm{Ohm}$ originally guessed as probable from the analogy of heat conduction, that this intensity is accurately proportional to the slope of potential which causes the flow; or, in other words (since action and reaction are equal and opposite), that a current in a conductor meets with an obstructive electromotive force exactly proportional to itself. The particular ratio between the two depends upon the particular material of which the conductor is composed, and is one of the constants of the material, to be determined by direct 